\title{
USO DE BIM PELOS PROFISSIONAIS DE ARQUITETURA EM CURITIBA
}

\author{
The use of BIM by architecture's professionals in Curitiba
}

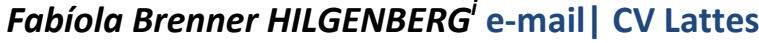 \\ Beatriz Lemos de ALMEIDA ii e-mail| CV Lattes \\ Sérgio SCHEER ${ }^{\text {iii }}$ e-mail | CV Lattes \\ Cervantes AYRES Filho ${ }^{\text {iv }}$ e-mail | CV Lattes
}

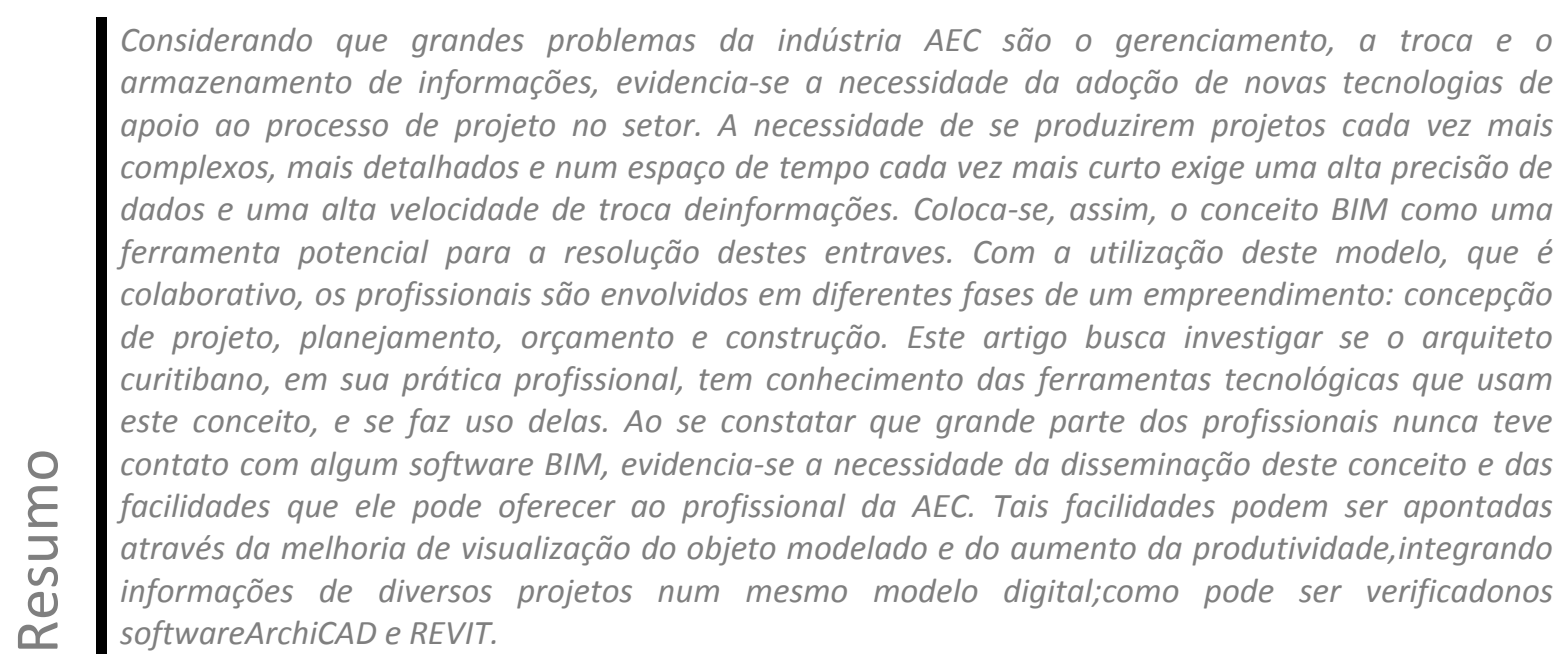

Palavras-chave: $A E C, B I M$, projeto.

Whereas the great problems of AEC industry are the management, exchange and storage of information, highlights the need to adopt new technologies to support the design process in the industry. The need to produce increasingly complex projects, more detailed and in an area of increasingly short time requires high precision and high speed data exchange information. There is, thus, the BIM concept as a potential tool for addressing these barriers. By using this model, which is collaborative, the professionals are involved in different phases of development: project design, planning, budgeting and construction. This paper investigates whether the architect of Curitiba, in his practice, knows the technology tools that use this concept, and makes use of them. When it appears that most professionals have never had any contact with BIM software, highlights the need for the dissemination of this concept and the facilities it can offer the professional AEC. Such facilities can be identified by improving visualization of the object modeled and increasing productivity, integrating information from multiple projects in a single digital model, as it can beverified in ArchiCAD and Revit software.

Palavras-chave: $A E C, B I M$, Design. 


\section{INTRODUÇÃO}

A necessidade da adoção de novas tecnologias de apoio ao processo de projeto no setor da Arquitetura, Engenharia e Construção é evidente. Grande parcela dos trabalhos científicos (AYRES, SCHEER, 2007; CRESPO, RUSCHEL, 2007a; GAO, MAHALINGAM, NGUTEN, 2008; EASTMAN etal, 2008; AZHAR, HEIN, SKETO; 2008) abordam problemas deste setor mencionando sua fragmentação, sua comunicação (interna e externa) deficiente, informalidade de troca de informações, diversidade de formatação de dados etc.

Nas últimas décadas, o processo de projeto arquitetônico vem sendo desenvolvido a partir dos mesmos métodos. Os desenhos, que eram realizados a mão, passaram a ser feitos nos computadores, com o uso de programas específicos que representassem virtualmente os projetos elaborados. Porém, há uma forte crítica sobre esta maneira de utilizar o computador: que não passaria de uma prancheta eletrônica e que, na realidade, seria um sub-uso das ferramentas que a informática pode disponibilizar (AYRES, SCHEER, 2007).

A necessidade de se produzirem projetos cada vez mais complexos, mais detalhados e num espaço de tempo cada vez mais curto exige uma alta precisão de dados e uma alta velocidade de troca de informações.

Os problemas da indústria AEC(Arquitetura, Engenharia e Construção) poderiam ser resolvidos com a adoção de um sistema eficaz de gerenciamento, troca e armazenamento de informações, dada as dimensões das equipes de projeto e a grande quantidade de fontes de informação, bem como de seu formato.

“0 desempenho de um projeto depende fortemente no quão bem a informação (...) é entregue e gerenciada. A conexão entre o projeto e a operação construtiva permanece baseada no papel ou em meios eletrônicos não-interativos. Este gargalo reduz significativamente a eficiência e a produtividade em campo." (GAO, MAHALINGAM, NGUTEN, 2008)

Neste contexto, surge o conceito BIM (Building Information Modeling).Para Crespo e Ruschel (2007a)a tecnologia BIM é um caminho para a representação do edifício virtualmente, onde objetos são codificados para representar elementos reais do ciclo de vida do edifício.

O ponto de partida é a concepção do projeto de um edifício, elaborada pelo arquiteto, pois é ele quem inicia o processo (CRESPO e RUSCHEL, 2007b). É nas fases iniciais da elaboração do projeto que surge a definição do software a ser utilizado, portanto é do arquiteto que deve partir a iniciativa de usar BIM. Por esta razão, este artigo pretendeu explorar o conhecimento destes profissionais a partir da elaboração e aplicação de um questionário dirigido a este grupo acerca do BIM.

\section{BUILDING INFORMATION MODELING}

O BIM (Building Information Modeling) é um conceito que dispõe de ferramentas tecnológicas para organização e gerenciamento da informação, utilizado durante todo o ciclo de vida de uma edificação: projeto, construção e demolição. Os softwares BIM possibilitam alterações dinâmicas no modelo e uma de suas inovações é a elaboração automática de layouts, cortes e elevações a partir de plantas (AYRES, AZUMA, SCHEER,2008). Além destes facilitadores, disponibilizam informações diretamente do projeto, como planilha orçamentária e especificações de materiais.

A probabilidade do modelo BIM ser caracterizado por informação inequívoca e consistente torna-se maior do que em CAD geométrico, pois representa a natureza do objeto (AYRES, SCHEER, 2007).

Com a utilização deste modelo, que é colaborativo, os profissionais são envolvidos em diferentes fases de um empreendimento: concepção de projeto, planejamento, orçamento e construção. Os programas BIM são responsáveis pela substituição do CAD geométrico por modelos 3D parametrizados. Com a difusão do uso no mercado de trabalho, aumenta a contribuição de demais profissionais. Para isto é necessário um software que suporte a colaboração de multiusuários com acesso simultâneo. 0 REVIT, por exemplo, comercializado pela Autodesk, possibilita esta interoperabilidade de dados. (COELHO, NOVAES, 2008) 
Além do aumento da produtividade, outros benefícios apresentados pelo BIM são a distribuição compartilhada de informações e a integração dos projetos em modelo único. Cabe ainda ressaltar que esta plataforma possibilita a ligação do objeto construído com as especificações técnicas relacionadas no site do fornecedor, a análise de compatibilidade construtiva entre os diferentes projetos, estudo de estimativa de custo e cálculo automático de áreas. Não se trata mais de desenhos desconectados meramente constituídos de linhas.

\subsection{Comparativo entre o CAD e o BIM}

A principal vantagem dos modelos de informação BIM é a consistência das informações, pois trata-se de um modelo virtual. Os arquivos CAD geométricos são formados por elemento geométricos básicos, sem qualquer referência, cabendo ao projetista interpretar e dar significado às linhas e demais elementos (AYRES e SCHEER, 2007).

Ao contrário dos programas convencionalmente utilizados (CAD geométricos), onde as alterações de projeto são corrigidas manualmente, os softwares BIM possibilitam alterações dinâmicas no modelo.A visualização das informações neste modelo se dá através de elementos tridimensionais, com modificações automáticas e interativas por alterações em qualquer uma das vistas. As informações no modelo BIM são centralizadas em um arquivo único, e o gerenciamento das mesmas é realizado através do software e não através de usuários.

Em relação a interoperabilidades os diferentes softwares CAD geométricos apresentam facilidades na transferência de arquivos.Os programas BIM, por ainda não serem amplamente difundidos no mercado, possuem restrições de acessibilidade e compatibilidade de arquivos com os programas mais antigos (SCHEER, S. et al, 2007).

0 principal obstáculo é em relação a complexidade dos programas BIM, em comparação com os CAD geométricos, pois apresenta grande número de parâmetros e exige conhecimento específico, tornando complexo o aprendizado dos novos usuários. Outra restrição é a não transferência das informações para formatos $d w g$ e similares (CRESPO, RUSCHEL, 2007b).

\subsection{Software BIM}

A utilização do BIM, pela AEC é apoiada pela nova geração de CADs. Os primeiros software BIM lançados no mercado foram o Allplan no início da década de 80 e o ArchiCAD, em 1984. 0 REVIT, outro software BIM, foi criado já na década de 90 , e posteriormente comprado e difundido comercialmente pela Autodesk.E entre outros, há também o software Bentley.

Estes programas que utilizam a tecnologia BIM, possuem inúmeras vantagens de visualização e melhoria na produtividade, em relação aos CAD geométricos mais difundidos no mercado, conforme descrito anteriormente. Alguns apresentam ainda a opção de importar arquivos dxf. para a compatibilização de projetos, reconhecendo também arquivos dwg (SCHEER, S. et al, 2007). Tais programas podem ainda contribuir integrando informações de diversos projetos em um único modelo digital. Este modelo é apoiado por um banco de dados composto de elementos construtivos e suas relações espaciais (FLORIO, 2007).

Sendo um dos últimos lançamentos da tecnologia BIM, o programa REVIT é composto pelo Revit Architecture, Revit Structure (Estrutura) e Revit System for mechanical, electrical and plumbing ${ }^{1}$ (MEP), capaz de partilhar um modelo único. Dentre estes programas, o RevitBuilding, mais voltado para a utilização com projetos, possui elementos construtivos (paredes, janelas, portas) com propriedades fixas (materiais, especificações), podendo o usuário alterar apenas valores determinados (dimensões). Sendo compartilhado por diversos usuários, um participante poderá propor alterações no modelo, que deverá ser conferido e validado pelos demais usuários antes de tornar-se uma alteração definitiva. Desta forma, a compatibilização dos projetos torna-se mais fácil e ocorre a redução de erros de projeto (CRESPO e RUSCHEL, 2007b).

O sistema é apresentado com informações em bancos locais, exclusivo para cada usuário do sistema. Estes usuários com acesso aos arquivos podem ser arquitetos, engenheiros, desenhista e qualquer outro profissional ligado ao processo de concepção do projeto. As alterações de arquivos são publicadas periodicamente no

1 Tradução livre: instalações prediais mecânicas, elétricas e hidráulicas 


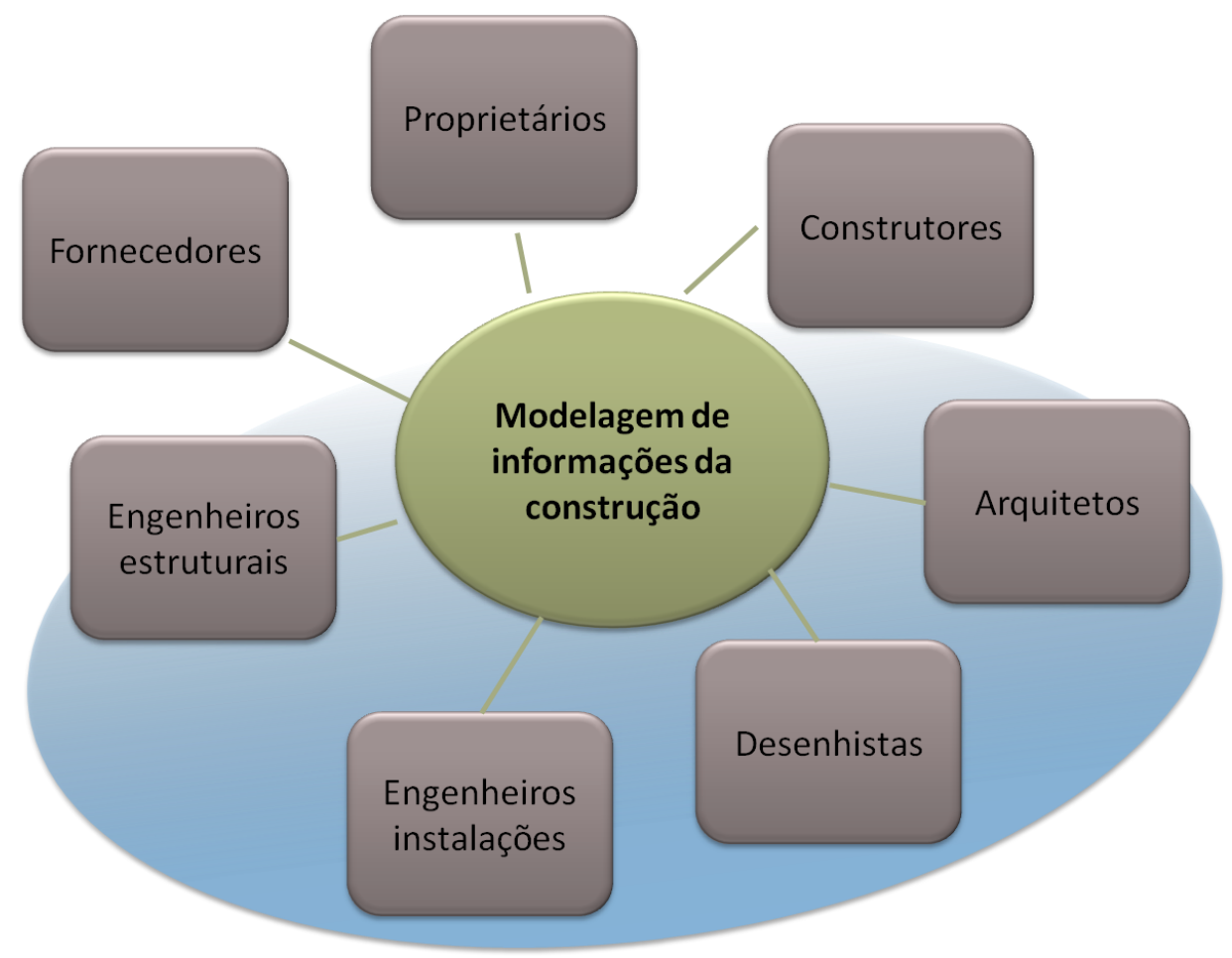

Figura 1 - Um modelo para todos os participantes do projeto. Fonte: Baseado em Autodesk.

Os programas que se utilizam da tecnologia BIM geram automaticamente além de cortes e elevações, tabelas de esquadrias, acabamentos e áreas, estimativa de cálculos e materiais, uma vez que os elementos inseridos são blocos e não apenas linhas. A partir disto, é capaz acompanhar o desenvolvimento de um projeto desde a fase de estudo preliminar, até a apresentação final com modelagem 3D. Outra melhoria apresentada é que usuários diversos podem utilizar um mesmo modelo, facilitando a compatibilização do projeto arquitetônico com projeto civil, hidráulico, estrutural e elétrico. Porém, alguns escritórios ainda utilizam programas de suporte para a geração de imagens.

O objetivo e benefício principal da utilização desta tecnologia é a integração de todos os dados multidisciplinares que compõe o projeto, além dos dados gerados em obra. A otimização de tempo e informações atinge também os orçamentistas do projeto, facilitando a compreensão e proporcionando uma visualização mais precisa dos materiais a ser quantificados (ALDER, 2006).

Podem-se listar ainda diversos atributos da ferramenta BIM que visam auxiliar nas estimativas de um projeto. Durante a criação de um modelo, automaticamente está disponível a lista de materiais associada ao modelo, além das dimensões e quantitativos destes materiais. È possível ainda isolar objetos na visão tridimensional para conferir a quantificação estipulado no orçamento, optando pela apresentação de detalhes significativos, complementando o entendimento do projeto. Após a alteração de partes do projeto ou materiais, é possível elaborar um comparativo entre os dados iniciais, através da tabulação de dados (ALDER, 2006).As principais vantagens apresentadas neste programas são a eliminação do re-trabalho e a melhoria na qualidade da informação disponibilizada.

\subsection{As barreiras para o uso de BIM no Brasil}

A transição de utilização no mercado deste novo modelo será gradual, em função das diversas barreiras existentes(AZHAR, HEIN, SKETO, 2008). Conforme já explicitado, a dificuldade de interoperabilidade com demais software utilizados no mercado ainda é um grande obstáculo para a difusão do BIM no contexto brasileiro. Os dados que alimentam os modelos devem ser constantemente atualizados pelos fornecedores da indústria AEC (IBRAHIM, 
KRAWCZYK, SCHIPPOREIT, 2004). Este fato representa uma dificuldade ainda maior no cenário nacional pelo restrito universo de usuários que aderiram ao sistema BIM e a consequente demanda limitada.

Para a implantação de software com conceito BIM, é necessária a presença de um hardware de alto desempenho e subsistemas de apoio. Além disso, para que seja difundido seu uso, o mercado precisa demonstrar maturidade organizacional e possuir metodologias de trabalho compatíveis. Segundo Nascimento e Santos (2002), uma das principais barreiras que impede o uso da tecnologia BIM é a resistência dos profissionais do setor da Construção Civil em relação às inovações. Além disso, as diferentes metodologias de trabalho e o uso de ferramentas diversificadas contribuem como mais um obstáculo de homogeneização de trabalho. Existe então a necessidade de melhorar a adequação do programa ao projeto, intensificar o treinamento e apoio técnico. Para transpor estes obstáculos, a mudança na prática arquitetônica deve prever a adequação da ferramenta proposta, utilizando todo o seu potencial (CRESPO, RUSCHEL, 2007b).

\section{MÉTODO DE TRABALHO}

Para a desenvolvimento deste trabalho usou-se o método de levantamento para coleta de dados através da pesquisa de campo. 0 instrumento escolhido para a coleta dos dados foi a aplicação de um questionário, utilizado então para gerar dados necessários para atingir o objetivo do projeto.

0 trabalho se estruturou em duas etapas, sendo a primeira uma revisão bibliográfica acerca dos conceitos de BIM (Building Information Modeling), além da análise de seu estágio de uso no Brasil.

Em seguida foi enviado o questionário paraa mailinglist do CREA, com6.200 profissionais. A proposta das perguntas iniciais deste questionário era realizar uma abordagem dos profissionais a partir da avaliação do conhecimento acerca deste conceito e da utilização dos softwares relacionados. Para a elaboração do questionário levou-se em consideração alguns componentes necessários para garantir a eficiência da pesquisa. Inicialmente foram identificados os respondentes através da seleção elaborada e a partir de então, enviado e-mail solicitando a colaboração na pesquisa através de uma prévia exposição do assunto e dos motivos que a motivaram. Além disso, foram realizadas as instruções sobre a forma de responder o questionário, com informações claras e objetivas.

Nas primeiras questões, utilizaram-se questões abertas, onde os respondentes ficam livres para empregarem suas próprias interpretações sobre o assunto. Segundo Mattar (1994) este tipo de questionário é vantajoso, pois estimula a cooperação, permite uma avaliação mais abrangente, tem menor poder de influência sobre as respostas dos colaboradores e proporciona comentários e esclarecimentos significativos sobre o assunto abordado. Porém, as pesquisa com questões abertas dão margem à parcialidade do entrevistador na compilação das respostas por não existir um padrão claro de respostas possíveis, tornando a interpretação subjetiva.

Com base nas perguntas iniciais foram acrescentadas outras questões, buscando investigar quais seriam as vantagens $\mathrm{e}$ as desvantagens para o profissional adotar um novo sistema de projeto. Nesta fase das perguntas, foram aplicadas questões de múltipla escolha, onde os entrevistados optaram por uma ou mais alternativas. Uma das considerações importantes para este tipo de formulário, é que as opções fornecidas devem cobrir todas as respostas possíveis. Porém, ao se incluir a alternativa "outros", garante-se a exclusão de alguma das opções. A facilidade de aplicação e da análise das respostas e o pequeno grau de erros são as principais vantagens deste questionário. De qualquer forma, este tipo de questionário exige um maior tempo de preparação de forma a garantir que o maior número de opções seja ofertado e ainda exerce uma influência direta no respondente de acordo com as alternativas apresentadas (MATTAR, 1994). 


\section{RESULTADOS}

\subsection{Perguntas iniciais: você conhece BIM?}

Ao constatar que a utilização do BIM deverá ser prevista nas fases iniciais da elaboração do projeto, momento em que o arquiteto coordena as atividades, iniciou-se esta pesquisa com um questionário voltado exclusivamente a este grupo de profissionais: arquitetos e engenheiros.

A seleção do grupo para responder à pesquisa partiu de um universo de arquitetos e engenheiros cadastrados no Conselho Regional de Arquitetura e Urbanismo do Paraná.Um questionário foi enviado por e-mail para 6.200 arquitetos e engenheiros no dia 30 de janeiro de 2012. Até o oitavo dia subsequente, foram obtidas 281 respostas, que representam $4,4 \%$ do total.Visando ampliar o universo de pesquisa, em
20 de fevereiro de 2012, foram enviadas novas solicitações de resposta ao questionário, igualmente aos 6.200 e-mails da lista. Nesta oportunidade, ampliou-se o total de resposta para 508, representando $8,2 \%$ do total. A partir disto, iniciou-se o trabalho de validação das respostas, utilizando-se como base a resposta dos 508 profissionais da área, sendo que94\% dos profissionais que responderam ao questionário eram arquitetos. Apenas 49\% responderam que possuíam conhecimento sobre o BIM, dos quais a maioria apresentou definições precisas acerca do conceito.

Quando questionados: "Você conhece o programa ArchiCAD?" 57\% afirmou que sim, embora somente $6 \%$ já tenha feito uso dele ao menos experimentalmente. Quanto ao software REVIT, 61\% dos profissionais disse conhecer, mas apenas $18 \%$ já fez uso.

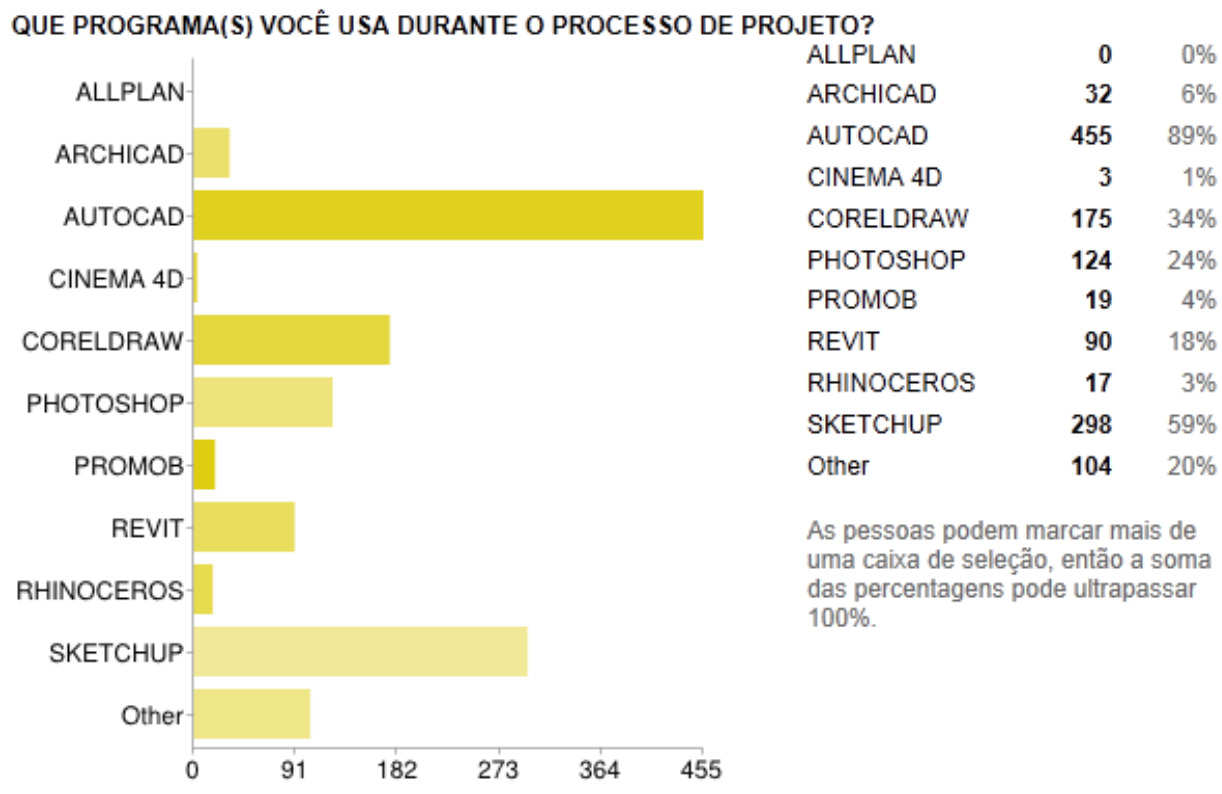

Gráfico 1 - Software usados pelos arquitetos para projetar.

Quando questionados a respeito de quais softwares utilizam para projetar, somente $6 \%$ usa ArchiCAD e $18 \%$ faz uso do REVIT, mas não exclusivamente, em ambos os casos.A maioria dos arquitetos não faz uso de software BIM. Conforme demonstrado no gráfico 1, 89\% dos arquitetos usam AutoCAD para projetar. O segundo software mais usado é o SketchUp, por $59 \%$ dos respondentes, sempre associado a outro programa. Em seguida, com $34 \%$ dos usuários, é o CorelDraw. Em quarto lugar, com porcentagem igual a $24 \%$, vêm o Photoshop e o
Revit na seqüência, com $18 \%$. Outros 28 software foram citados, por $16 \%$ dos arquitetos, sendo os programas VectorWorks e 3ds Max (3D Studio Max) utilizados por 3,5\% dos respondentes, cada um.

Somente $49 \%$ dos profissionais já tiveram algum contato com software BIM. Do total dos entrevistados, apenas $29 \%$ utilizam alguns dos softwares que suportam a tecnologiaBIM para projetar, que segundo Faria (2007) são o ArchiCad, Revit, Bentlay e Vector Works. 


\subsection{Questões de múltipla escolha: o que faria você adotar um novo software?}

Com a definição das perguntas finais do questionário procurou-se saber quais seriam as principais vantagens e as principais desvantagens para um profissional adotar ou não um novo software para projetar.

0 principal atrativo, citado por $74 \%$ dos profissionais, seria a agilidade nas modificações de projeto. A facilidade de compatibilização com programas de projetos complementares é o segundo fator mais relevante para incentivar a mudança, considerado por $73 \%$. A interoperabilidade com outros programas, a geração automática de planilhas quantitativas e orçamentárias e facilidade de visualização pelo cliente são consideradas importantes por mais da metade dos arquitetos. Ver gráfico 2 .

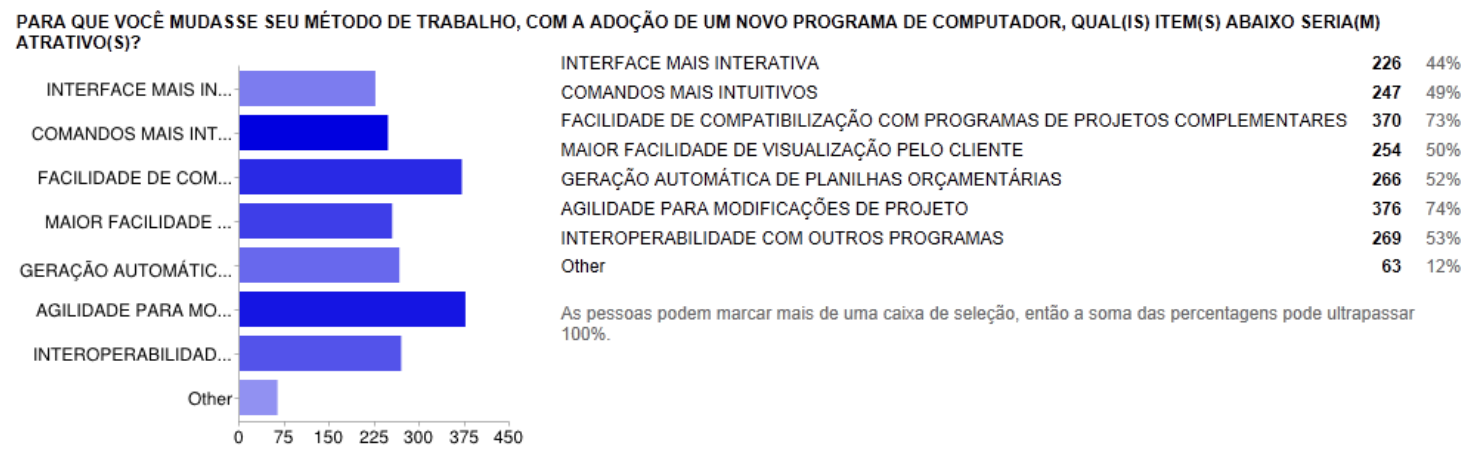

Gráfico 2 - Atrativos para mudar de software.

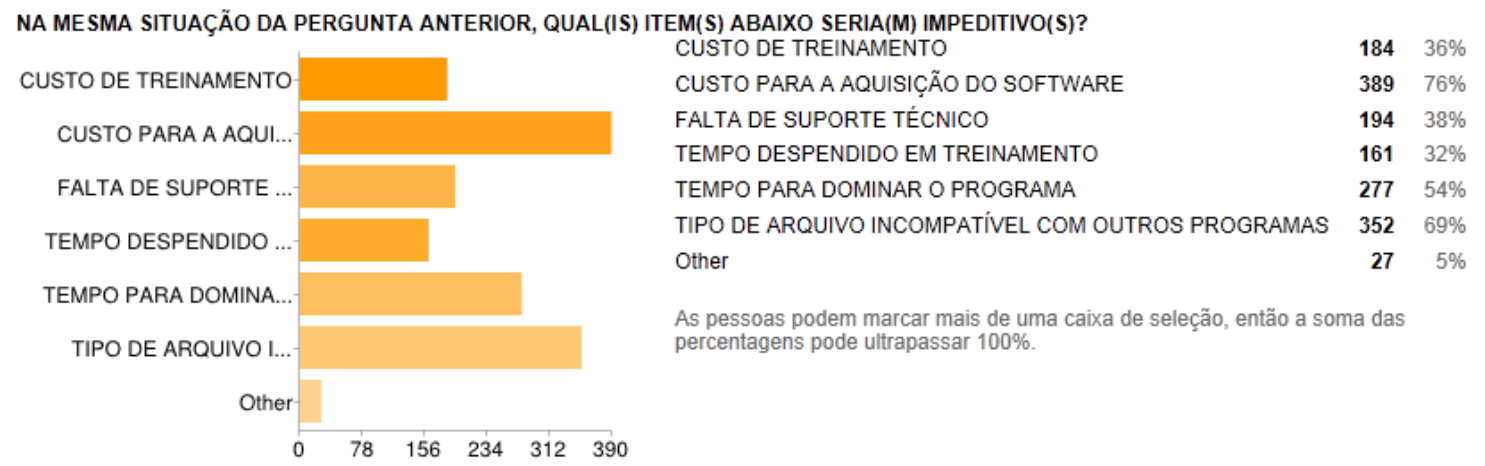

Gráfico 3 - Impeditivos para mudar de software.

O item considerado como maior impeditivo para a mudança de software é o custo para a aquisição do software, segundo $76 \%$ dos profissionais. $69 \%$ considera o tipo de arquivo incompatível com outros programas uma barreira e mais da metade dos arquitetos julga impeditivo o tempo para dominá-lo. Ver gráfico 3.

Os fatores menos relevantes seriam o custo e otempo despendido em treinamentos, apontados por apenas um terço dos profissionais. E somente $38 \%$ se incomodariam com a falta de suporte técnico.
5\% dos profissionais apresentaram outros impeditivos para a adoção de diferentes softwares, argumentaram a relevância de uma ação de mudança coletiva. É de extrema importância que todos os colaboradores e projetistas envolvidos no processo tenham adotado a mesma linguagem de trabalho, no caso, BIM. Um esforço individual teria resultados muito limitados, dificultando a comunicação pela falta de migração dos demais envolvidos no processo. 


\section{DISCUSSÃO}

A partir dos dados levantados na primeira rodada, constata-se que há necessidade que os software BIM sejam mais difundidos, pois mais da metade dos profissionais da amostra nunca teve qualquer contato com tais ferramentas.

0 verdadeiro potencial da tecnologia e o domínio do conceito BIM são consequências de seu uso, o principal quantificador é o fato dos profissionais não terem contato com estas ferramentas. 0 conceito seria relevante como publicidade para o incentivo de seu uso.

A maior barreira para a adoção de um novo software, segundo os arquitetos questionados, seria a incompatibilidade de arquivos com outros programas, o que é o caso, por exemplo, dos arquivos REVIT.Contudo, apenas após um treinamento apropriado e consequente domínio do programa, os software complementares serão dispensáveis.

A maioria dos profissionais não vê o tempo e os recursos despendidos em treinamento como impedimentos para a troca de programa, mas se preocupa com o tempo que se leva para dominálo.

\section{CONCLUSÕES}

Os arquitetos questionados são favoráveis à adoção do BIM. Quase todos estariam dispostos a adotar um software que otimizasse seu tempo, tanto no momento da concepção do projeto quanto na sua compatibilização com projetos complementares e possíveis modificações.

0 início desta mudança seria a divulgação do conceito BIM, para que o profissional tome conhecimento das possibilidades que esta nova tecnologia pode oferecer. A observação mais importante feita nos questionários é o fato de que esforços individuais não chegam a atingir o principal objetivo do BIM, que é a integração absoluta do projeto e dos membros de suas equipes com o mundo real. 0 importante é uma sensibilização coletiva para adoção de sistemas compatíveis.

\section{TRABALHOS FUTUROS}

A partir dos dados levantados, constatou-se a necessidade da disseminação do conceito BIM. Para tanto, está elaborado um quadro informativo sobre o conceito e as facilidades que
BIM oferece para os profissionais da área de AEC.Este quadro será enviado por e-mail para os participantes da pesquisa e outros arquitetos e engenheiros.

\section{REFERÊNCIAS}

ALDER, M. Adam. Comparing time and accuracy of building information modeling to onscreen take off for a quantity takeoff on a conceptual estimate. Dissertação (Master of Science) School of Technology Brigham Young University, $\quad$ Estados 2006. http://contentdm.lib.byu.edu/cdm/singleitem/id/729/collection/ETD

AYRES Filho, Cervantes, SCHEER, Sergio. Diferentes abordagens do uso do CAD no processo de projeto arquitetônico. In: Anais do VII Workshop Nacional de Gestão do Processo de Projeto na Construção de Edifícios, Curitiba, 2007.

AYRES Filho, C.; AZUMA, F. e SCHEER, S.; Utilização do CAD-BIM para projetos de alvenaria de blocos de concreto. 2008. In: Anais do VIII Workshop Nacional de Gestão do Processo de Projeto na Construção de Edifícios, São Paulo, 2008.

AZHAR , Salman, HEIN, Michael; SKETO, Blake. Building Information Modeling (BIM): Benefits, Risks and Challenges. Proceedings of the 44th ASC National Conference, 2008 
COELHO, S. S. e NOVAES, C. C.; Modelagem de Informações para Construção (BIM) e ambientes colaborativos para gestão de projetos na construção civil. In: Anais do VIII Workshop Nacional de Gestão do Processo de Projeto na Construção de Edifícios, São Paulo, 2008.

CRESPO, C. e RUSCHEL, R. C.; Solução BIM para a melhoria no processo de projetos. V SIBRAGEC. Campinas: 2007a.

.; Ferramentas BIM: Um desafio para a melhoria no ciclo de vida do projeto. TIC 2007.Porto Alegre: $2007 \mathrm{~b}$

EASTMAN, Chuck; TEICHOLZ, Paul; SACKS, Rafael; LISTON, Kathleen. BIM Handbook: A guide to Building Information Modeling for Owners, Managers,Designers, Engineers, and Contractors. New Jersey: John Wiley\& Sons, Inc., 2008.

FARIA, Renato. Construção Integrada. Revista Téchne. Edição 127, 2007.

FLORIO, Wilson. Tecnologia da informação na construção civil: contribuições do Building Information Modeling no processo de projeto em arquitetura. III Fórum de Pesquisa FAU MACKENZIE, São Paulo, 2007.

GAO, Z.; MAHALINGAM, G. e NGUTEN, T. Applications or Building Information Modeling (BIM) in the Design and Construction Process. ICCCBE. China: 2008.

IBRAHIM, M.; KRAWCZYK, R.; SCHIPPOREIT, G.Two approaches to BIM: A Comparative Study. eCAADe Conference. Kopenhagen, Dinamarca, 2004.

MATTAR, Fauze Najib. Pesquisa de marketing: metodologia, planejamento, execução e análise. São Paulo: Atlas, 1994.

NASCIMENTO, Luiz Antonio; SANTOS, Eduardo Toledo. Barreiras para o uso da tecnologia da informação na indústria da construção civil. In: Anais do II Workshop Nacional de Gestão do Processo de Projeto na Construção de Edifícios, Porto Alegre, 2002.

SCHEER, S.; ITO, A. L. Y.; AYRES Filho, C.; AZUMA, F. e BEBER, M.; Impactos do uso do sistema CAD geométrico e do uso do sistema CAD-BIM no processo de projeto em escritórios de arquitetura. In: Anais do VII Workshop Nacional de Gestão do Processo de Projeto na Construção de Edifícios, Curitiba, 2007. 


\section{APÊNDICE- QUESTIONÁRIOS}

\section{PRIMEIRA RODADA: VOCÊ CONHECE BIM?}

Nome

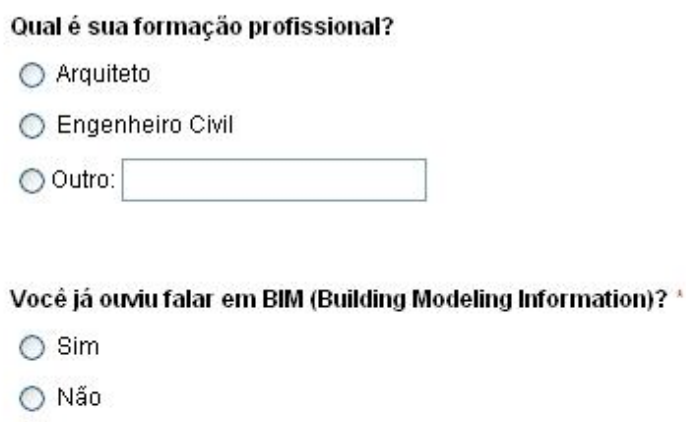




\title{
II. SEGUNDA RODADA: O QUE FARIA VOCÊ ADOTAR UM NOVO SOFTWARE?
}

\author{
Nome \\ $\square$ interface mais interativa \\ $\square$ comandos mais intuitivos \\ facilidade de compatibilizaçẫo de projetos complementares \\ maior facilidade de visualizaçẫo pelo cliente \\ maior eficiência (reduçẫo do tempo de trabalho pelo mesmo resultado) \\ geração automática de planilhas quantitativas e orçamentárias \\ agilidade para modificaçỗes no projeto \\ interoperabilidade outros programas \\ Doutro: \\ Na mesma situação, qual(is) do(s) item(s) abaixo seria(m) impeditivos? \\ $\square$ tempo despendido em treinamento \\ custo para a aquisiçã̃o do software \\ custo de treinamento \\ falta de suporte técnico \\ tempo para dominar o programa \\ tipo de arquivo incompativel com outros programas \\ Outro:
}

Para que você mudasse seu método de trabalho (com a utilizaçäo de um novo software), qual(is) do(s) item(s) abaixo seria(m) atrativo(s)?

\section{DADOS DOS AUTORES}

(i) Professora da Universidade Positivo

(ii)

(iii) Professor Associado da Universidade Federal do Paraná.

(iv) Graduado em Arquitetura e Urbanismo pela Universidade Federal do Paraná (2005). Mestrado em Construção Civil no PPGCC-UFPR.. COMPLETAR COM ENDERE"CO PARA CORRESPONDENCIA EXIGENCIA DAS BASES DE INDEXAÇÃO 